\title{
Deep mixed model for marginal epistasis detection and population stratification correction in genome-wide association studies
}

\author{
Haohan Wang ${ }^{1}$ (D), Tianwei Yue ${ }^{2}$, Jingkang Yang ${ }^{3}$, Wei Wu ${ }^{4}$ and Eric P. Xing ${ }^{5^{*}}$ \\ From Joint 30th International Conference on Genome Informatics (GIW) \& Australian Bioinformatics and Computational \\ Biology Society (ABACBS) Annual Conference
}

Sydney, Australia. 9-11 December 2019

\begin{abstract}
Background: Genome-wide Association Studies (GWAS) have contributed to unraveling associations between genetic variants in the human genome and complex traits for more than a decade. While many works have been invented as follow-ups to detect interactions between SNPs, epistasis are still yet to be modeled and discovered more thoroughly.

Results: In this paper, following the previous study of detecting marginal epistasis signals, and motivated by the universal approximation power of deep learning, we propose a neural network method that can potentially model arbitrary interactions between SNPs in genetic association studies as an extension to the mixed models in correcting confounding factors. Our method, namely Deep Mixed Model, consists of two components: 1) a confounding factor correction component, which is a large-kernel convolution neural network that focuses on calibrating the residual phenotypes by removing factors such as population stratification, and 2) a fixed-effect estimation component, which mainly consists of an Long-short Term Memory (LSTM) model that estimates the association effect size of SNPs with the residual phenotype.

Conclusions: After validating the performance of our method using simulation experiments, we further apply it to Alzheimer's disease data sets. Our results help gain some explorative understandings of the genetic architecture of Alzheimer's disease.
\end{abstract}

Keywords: Marginal epistasis, Mixed model, GWAS, Deep learning

\section{Background}

Genome-Wide Association Studies (GWASs) have helped uncover associations between genetic variants and complex traits for more than a decade. The methods for GWA studies first started with the univariate hypothesis testing, and later, many advanced statistical and machine learning methods have been proposed to infer and gain

\footnotetext{
*Correspondence: epxing@cs.cmu.edu

${ }^{5}$ Machine Learning Department, School of Computer Science, Carnegie Mellon University, Pittsburgh, PA, USA

Full list of author information is available at the end of the article
}

insights into the genetic architectures of the complex traits. For example, linear mixed models are demonstrated with empirical successes in correcting confounding factors raised by population stratification, family relatedness, and cryptic relatedness [1-5], and multivariate regression methods are introduced for modeling the polygenetic effects [6-8]. Integration of these two methods is also introduced to successfully consider polygenicity and confounding factor correction together $[9,10]$.

Despite promising results have been generated using these approaches, it has been long known that additive

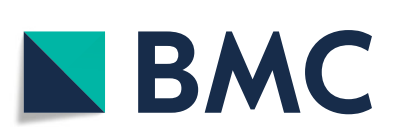

(c) The Author(s). 2019 Open Access This article is distributed under the terms of the Creative Commons Attribution 4.0 International License (http://creativecommons.org/licenses/by/4.0/), which permits unrestricted use, distribution, and reproduction in any medium, provided you give appropriate credit to the original author(s) and the source, provide a link to the Creative Commons license, and indicate if changes were made. The Creative Commons Public Domain Dedication waiver (http://creativecommons.org/publicdomain/zero/1.0/) applies to the data made available in this article, unless otherwise stated. 
effects can explain only part of genetic variations [11]. Epistasis (i.e., interactions between genetic variants) is believed to be a potential source of the unexplained variations [12-15]. Evidence of epistatic interactions has been shown for human complex traits [16-18], suggesting that more potential interactions between genetic variants are to be discovered, which motivates the development of more powerful computational methods.

Epistasis detection is usually highly computational challenging, and thus many efforts have been made by gearing towards developing efficient computational tools for discovering epistasis with different searching strategies, including exhaustive [19-23], probabilistic [24], or prioritized search [25-30]. In addition to these methods that mainly focus on the detection of pairwise interactions of SNPs, a few methods were developed for detecting higher order interactions, and they either rely on probabilistic sampling [31] or ultra-high-performance computing service [32]. Recently, Crawford et al proposed an alternative strategy for testing the exact combinations of candidate SNPs. Their method, named MAPIT, tests to identify the SNPs that involved in the epistasis marginally [33]; in other words, their aim to identify the SNPs that are associated with the phenotype in an epistastic manner without revealing the exact combination of these SNPs.

In this paper, continuing with the goal of investigating marginal epistasis, we propose a deep-learning-based method that can implicitly model arbitrary high-order interactions between genetic variants, as well as simultaneously correct confounding effect due to population stratification, family structure, and cryptic relatedness. The central design rationale behind our model is the universal approximation property of deep neural networks [34], which allows neural networks to model arbitrary interactions of the input features (i.e., epistasis). To take advantage of this property, we propose the Deep Mixed Model (DMM). DMM consists of two components: 1) A confounding factor correction component that is a onedimensional convolutional neural network $(\mathrm{CNN})$ with a large kernel size, thus CNN can focus mostly on the population-wise pattern of data. 2) A variable selection component that mainly consists of a fine-grained Longshort Term Memory (LSTM) model with sparse variable selection methods plugged in; this component is responsible for identifying the SNPs that are associated with the residual phenotype in univariate, polygenetic, or epistastic manners.

We first conduct simulation experiments to demonstrate the superior empirical performance of DMM over competing methods and to inspect and verify the internal working mechanism of DMM. Then we apply DMM to real-world Alzheimer's disease data sets, and DMM identifies several interesting SNPs. Some of these results are supported through literature surveys, which suggest that our findings, despite explorative at the current stage, may lead to some novel understandings of the Alzheimer's disease.

\section{Methods}

In this section, we formally introduce our proposed Deep Mixed Model, which is composed of two components, one for confounding factor correction and the other for genetic variants selection. We refer to these two components as corrector and selector for convenience. We first present the overall concept and then discuss each component in detail.

\section{Overview}

Figure 1 illustrates the main idea of our proposed Deep Mixed Model, which consists of two components: 1) the red part of the figure represents the corrector, which is a convolutional neural network with a large kernel size. The large kernel size forces the $\mathrm{CNN}$ to focus more on the overall pattern represented by the genetic variants, instead of variations of specific SNPs, and thus resulting in a population effect estimator; and 2) the blue part of the figure represents the selector, which is an LSTM with a sparse vector attached at the input. We will discuss the details of these two components immediately after this overview.

In this paper, we use $\mathbf{X} \in \mathcal{R}^{n \times p}$ to denote the SNP array in our study, $\mathbf{y} \in \mathcal{R}^{n \times 1}$ to denote the phenotype, where $n$ represents the number of samples, and $p$ represents the number of SNPs. We use $\beta$ to denote effect sizes for fixed effects and $\mathbf{u}$ to denote effect sizes for random effects. The dimension of $\beta$ and $\mathbf{u}$ can be inferred from the context. We use $f(\cdot ; \delta)$ to denote the corrector, and $\delta$ stands for the corresponding parameters. Similarly, we use $h(\cdot ; \theta)$ to denote the selector, and $\theta$ stands for the parameters. $g^{-1}(\cdot)$ denotes the inverse linkage function of a generalized linear model. $\epsilon$ denotes natural noise which is negligible in most cases throughout this paper.

\section{The confounding factor correction component (the corrector)}

To account for confounding factors, we propose a one-dimensional convolutional neural network that estimates the population-level effects and further calculates the residual phenotype after removing these effects. To enforce that CNN primarily focuses on estimating population-level effects, we adopt a large size of the convolutional kernel, based on the understanding that a kernel with large size will encourage the network to learn high-level conceptual representations - rather than detailed variations - of the data [35]. Different from the conventional mixed models that estimate the secondorder statistics (variance) raised by confounding factors using the kinship matrix [36], the corrector directly operates on the data matrix and estimates the first-order 


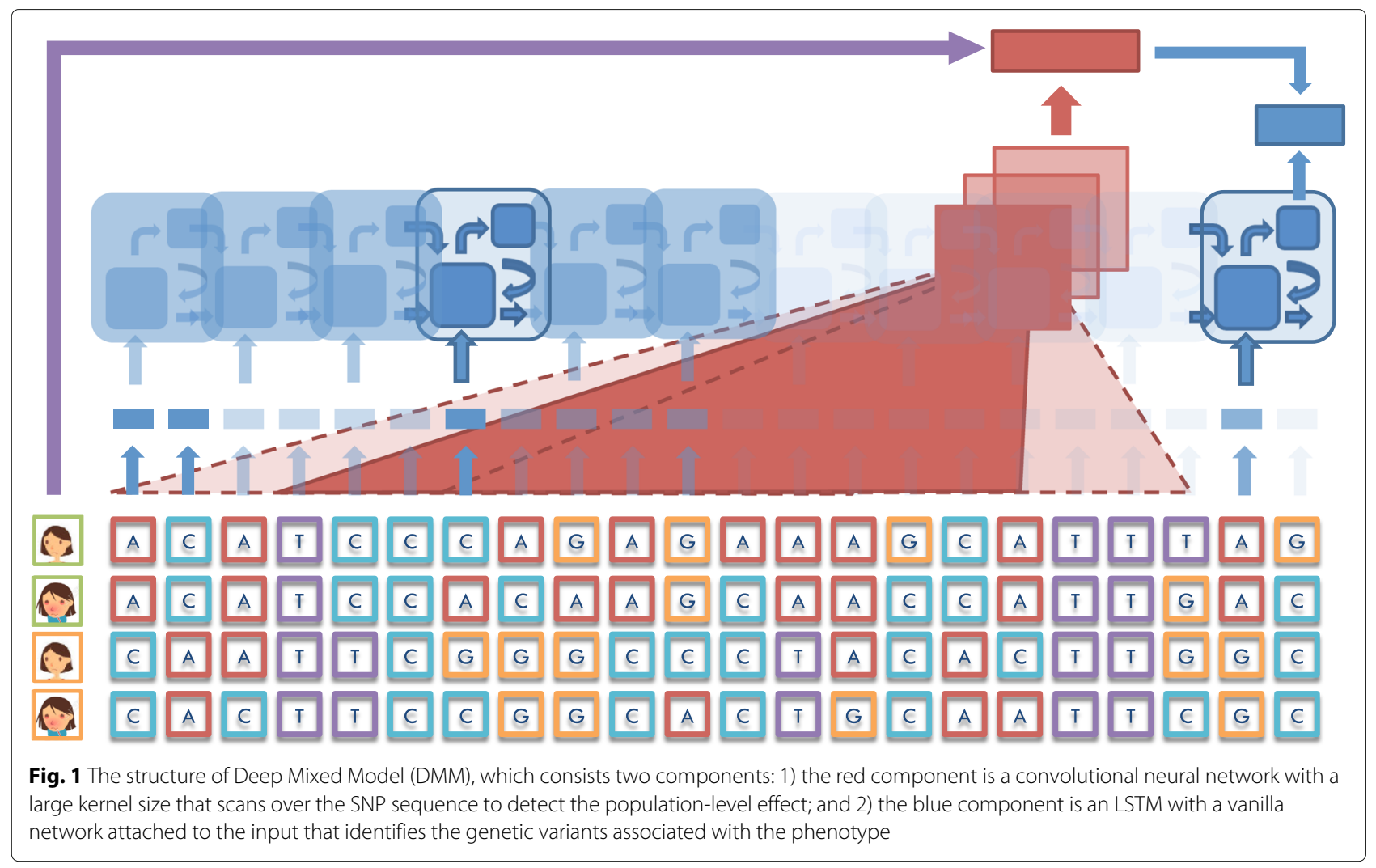

statistics, which is also sufficient in helping remove the confounding factors, justified by the resemblance between a linear mixed model and a ridge regression (Wang $\mathrm{H}$, Aragam B, Xing EP: Statistical analysis of linear mixed model for gwas. in preparation).

\section{The fixed-effect estimation component (the selector)}

For the component that is responsible for selection of genetic variants, we choose the LSTM. Instead of feeding the data directly into the LSTM, we add a one-dimension weighing vector for SNPs; by doing so, the magnitude of the corresponding value of the weighting vector can directly reflect the importance of the genetic variants evaluated by the model, as shown by [37]. More specifically, we can decompose the selector as:

$$
h\left(\mathbf{X}_{i} ; \theta\right)=l\left(\mathbf{X}_{i} \odot \omega ; \iota\right)
$$

for $i^{\text {th }}$ sample, where $\odot$ denotes element-wise product, $\omega$ denotes the weighting vector, and $l(\cdot ; \iota)$ denotes the generic LSTM module whose parameters are denoted as $\iota$. The fixed-effect estimation component consists of both $\omega$ and $l(\cdot ; \iota)$, and we denote the parameters as $\theta=[\omega ; \iota]$.

\section{Algorithm}

The algorithm for solving DMM splits into two steps: 1$)$ estimating the parameter $\delta$ for the corrector $(f(\cdot ; \delta))$, and 2) estimating the parameter $\theta$ for the selector $(h(\cdot ; \theta))$.
The estimation of $\delta$ can be done straightforwardly by solving:

$$
\hat{\delta}=\underset{\delta}{\arg \min } c(\mathbf{y}, f(\mathbf{X} ; \delta))
$$

where $c(\cdot, \cdot)$ is a generic cost function; for example, we can use the mean squared loss for data with continuous phenotypes and use the cross entropy loss for case-control data.

With $\hat{\delta}$, we can further estimate $\theta$ by solving:

$$
\hat{\theta}=\underset{\theta}{\arg \min } c\left(\mathbf{y}, g^{-1}(h(f(\mathbf{X} ; \hat{\delta}) ; \theta))\right)
$$

where $g(\cdot)$ can also be chosen based on the understanding of data; for example, a linear function can be used for continuous phenotypic data and a logic function for case-control data.

It is essential to avoid overfitting in genetic studies, especially because the psychiatric genetic data are costly to obtain, and we usually only have a sample size of a couple hundred. To avoid overfitting, we stop the training process before the optimization starts to converge, which is known as early-stopping, a regularization method for neural networks $[38,39]$. While both Function 1 and Function 2 are optimized with early-stopping, we empirically notice that, in the simulation experiments, the earlystopping is particularly crucial for optimizing corrector since it effectively prevents the $\mathrm{CNN}$ from estimating 
additional (unnecessary) information other than true confounding effects from population-level factors. We notice that the corrector only needs to be tuned for about 10 epoches.

The detailed configurations of our method mentioned above are summarized in Table 1. With such configuration, in practice, it takes our method less than an hour to converge on the real data experiment (details to be followed in the "Results" section) with a modern GPU. Our method scales well with the number of samples, but limited with the number of SNPs considered due to the limitation of the memory of GPU or CPU.

\section{Results}

In this section, we will introduce our experiment results, including the simulation results where we compare our method with competing methods and the findings when we apply the DMM to real data. The TensorFlow experiment scripts to replicate the results are submitted as the Supplement. We also released our script as a tool for the community to apply on other data sets at: https://github.com/HaohanWang/DMM.

\section{Simulations}

\section{Competing methods}

To evaluate the performance of DMM, we compare it with several existing methods listed as follow:

- UT: The standard univariate testing (Wald testing) with the Benjamini-Hochberg (BH) procedure [40]. This is the most popular approach for testing associations in GWAS, without concerning epistasis or accounting for population stratification.

- LMM: A standard linear mixed model with the BH procedure. This is the most popular approach in GWAS for handling population stratification, but not concerning epistasis.
- Lasso: The $\ell_{1}$-regularized linear regression [41]

- Adaptive Lasso (AL): An extension of Lasso that weighs the regularization term accordingly [7] (enabled by the method introduced in [42] for high-dimensional data).

- Precision Lasso (PL): A novel variant of Lasso that can handle correlated and linearly dependent features commonly used in genomics study [8].

- MAPIT: The marginal epistasis test, a method recently proposed for detecting epistasis in GWAS [33]. We re-implement the method in Python for fair comparison. We also add the $\mathrm{BH}$ procedure [40] for false discovery control.

- LSTM: The selector in the Deep Mixed Model. We test the performance of this component of DMM without the confounding factor correction component.

- DMM: The method we proposed in this paper. ROC curve is calculated with different thresholds of absolute effect sizes.

\section{Data generation}

We use SimPop [43] to simulate the SNP array. We simulate $p=10000$ SNPs for $n=500$ or 1000 samples from five different populations with migration behaviors. Each population also unevenly splits into five sub-populations. Therefore, it can be seen as these samples are from 25 regions (denoted as $\mathbf{G}$ ) out of five continents. As we mentioned previously, the SNP array is denoted as $\mathbf{X}$. We choose the number of samples to be small to reflect the situation of our real psychiatric data.

We select $k$ SNPs to be associated with the phenotype, and to simulate the arbitrary interaction patterns of these SNPs, we set a group size of $t$ to group these $k$ SNPs into $m$ groups (the number of groups $m=k / t$, where $k$ is divisible by $t$ ), and sample $m$ effect sizes: each of them is sample as $\beta \sim N(0,25)$ (This value of variance is

Table 1 Detailed configurations of the method

\begin{tabular}{llll}
\hline Collector (1D-CNN) & Convolutional layer & Num. of Kernels: 16 & Kernel Size: $1000 \times 1$ \\
& Pooling layer & Initializer: Truncated normal initializer & Padding: Same \\
& 1st fully-connected layer & Size: 2000 & Stride: 2000 \\
& 2nd Fully-connected layer & Output: 1 & Dropout rate: 0.9 \\
& Weighting layer & Num. of units: $p$ (one-to-one layer) \\
Selector (LSTM) & Hidden layer & Num. of units: $0.15 p$ & \\
Optimizer (ADAM) & Learning rate: 0.001 & Batch size: 128 & \\
Other hyperparams & Collector's epoch: 20 & Selector's epoch: 1500 & \\
\hline
\end{tabular}

The architecture and hyperparameters are selected through the experiments with simulated data, and are used without changes for real data experiments 
chosen following the suggestion of [44] as an intermediate effect size).

As we mentioned previously in the Introduction, there are plenty of methods that can identify the SNPs that are associated to the phenotype with lower order of interaction manner. Therefore, in the experiment, we focus on experimenting with the remaining situation when the multiple SNPs interact $(t=5)$, which is more challenging than usual epistasis experiment set-up. However, our set-up is not contradictive to the real-world setting, as this remaining situation will be met when we regress out the lower-order SNP effects.

To introduce confounders such as population stratification and family structure, we use the regions $\mathbf{G}$ to affect the phenotypes differently (the effects of these regions are denoted as $\gamma$, sampled from a Gaussian distribution $\left.N\left(0, \sigma_{u}^{2}\right)\right)$. The variation of $\sigma_{u}^{2}$ results in a signal-to-noise ratio of 0.25 or 1.0 for $\beta$ in our simulation experiment.

Finally, we have the responses as:

$$
\mathbf{r}=\sum_{i=1}^{m}\left(\prod_{j \in i} \mathbf{X}_{j}\right) \beta_{i}+\mathbf{G} \gamma
$$

where we use the product sign (П) to denote the interaction of the SNPs. We use the element-wise minimum to simulate the interaction. $j \in i$ denotes that the SNP (indexed by $j$ ) out of the $k$ associated SNPs that belong to the group $m$. We test the methods with the continuous phenotypes generated as

$$
\mathbf{y}_{c}=\mathbf{r}+\epsilon,
$$

where $\epsilon \sim N(0,1)$. Additionally, we also transform these continuous responses $r$ into binary phenotypes via Bernoulli sampling with the outcome of the inverse logit function $\left(g^{-1}(\cdot)\right)$ over current responses. Therefore, we have:

$$
\mathbf{y}_{b}=\operatorname{Ber}\left(g^{-1}(\mathbf{r})\right)
$$

We experiment on both continuous data $\mathbf{y}_{c}$ and binary data $\mathbf{y}_{b}$. The main steps of this simulation data generation process are conveniently illustrated by Figure 2. Due to the introduction of epistasis, our simulation data becomes extremely difficult for conventional methods to recover the signals, as we will show in the next section.

\section{Main simulation results}

We test the methods with different settings of different number of samples $n \in\{500,1000\}$ of the effects from confounders $\sigma_{u}^{2} \in\{5,10\}$, the number of associated SNPs $k \in\{10,50\}$, and for continuous phenotype $y_{c}$ and binary phenotype $y_{b}$ respectively. There all together 16 different experimental settings, and we run 20 different seeds of each setting. In all these experiments, we investigate the results for the SNPs that are ranked in the first 1000 associated SNPs. Because of the difficulty of our simulation set-up, almost no methods can report meaningful results within top 100 or less reported SNPs.

We evaluate these methods with ROC curves. For testing-based methods (UT, LMM, MAPIT), the ROC

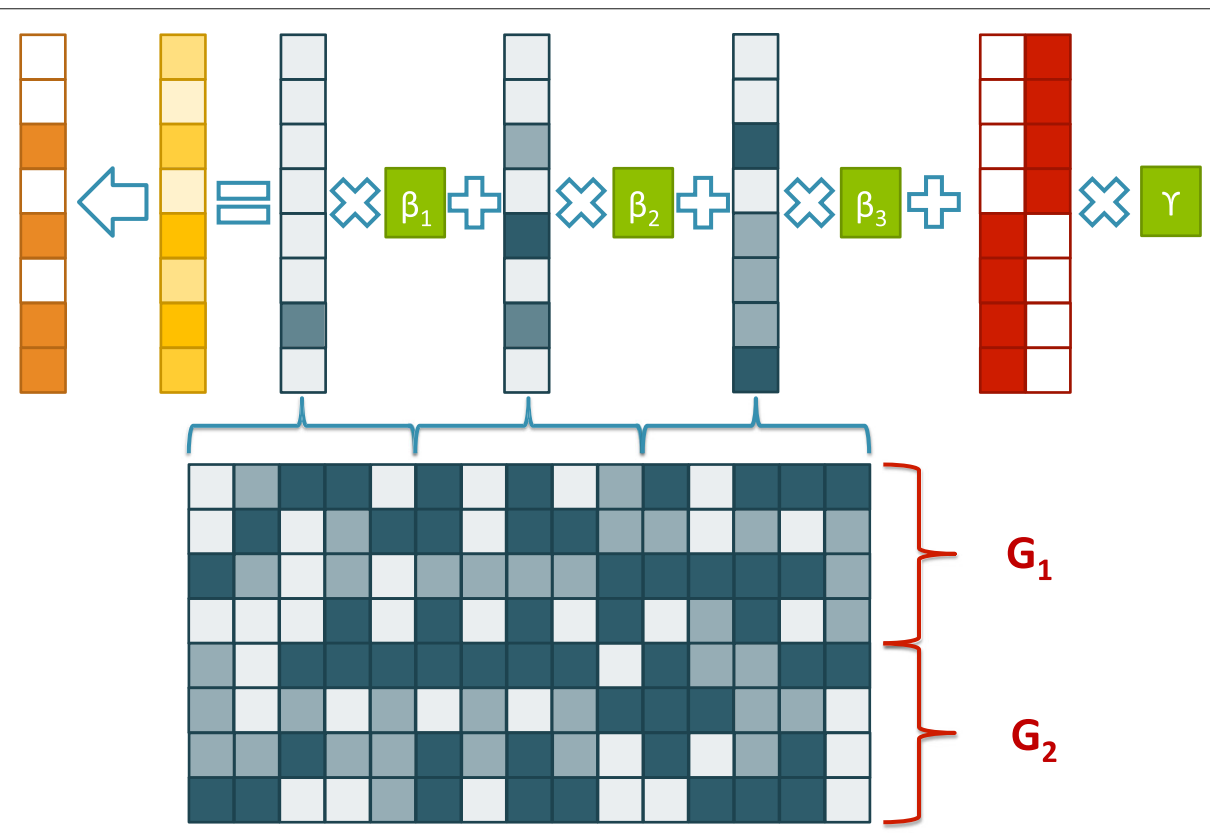

Fig. 2 Illustration of the main steps of the simulation data generation process. The dark squares represent the SNP array, with two populations (marked with red descriptions). We group every five SNPs and simulate their interaction, result in one epistatic variable. For each epistatic variable, we introduce an effect size. Summing over the effects introduced by these epistatic variable, together with the effects introduced by population structure, we result in an continuous variable, which will further be transformed into binary phenotype 
curve is plotted by variation of the threshold of $p$-values. For multivariate regularized methods (Lasso, AL, PL), the ROC curve is plotted with hyperparameters (regularization weight) varying evenly in the logspace from $10^{-5}$ to $10^{5}$. For deep learning methods, the ROC curve is plotted with different thresholding of absolute value of estimated selector parameter $\omega$.

Figure 3 shows the simulation results. As we can see, our proposed DMM method has a clear advantage over the competing methods. We can see that almost all the regularized multivariate regression method (Lasso, AL, PL) behave unsatisfyingly in these simulations. We believe this is because of the effects introduced from the confounders. Interestingly, vanilla Wald test generally behave better than other methods despite that it considers neither epistatic effects (not even multivariate effect) nor confounding factors.

By comparing the results in continuous case and the corresponding results in binary case, all these methods behave better in continuous case than in binary case. This is expected because continuous response contains more information. By comparing different settings, the experimental results of methods behave as expected: with less confounding effects, and more samples, the experimental results tend to be better. Also, interestingly, we notice that these methods tend to behave better when there are less associated SNPs to be tested.

To have a more detailed comparison, we also study the averaged Area under ROC of different settings of the experiments corresponding to the results Fig. 3 shows, details shown in Table 2. Notice that all these methods only select top 10\% (1000 SNPs) as candidate SNPs for plotting ROC and calculating AUC, which is the primary reason that the regularized multivariate regression method shows a result of exactly 0.5 .

When the phenotype is continuous, DMM shows a clear advantage over other methods, while the LSTM follows in the second place. Therefore, we can safely draw the conclusion that the differences between DMM and the LSTM are due to the ability of the corrector for

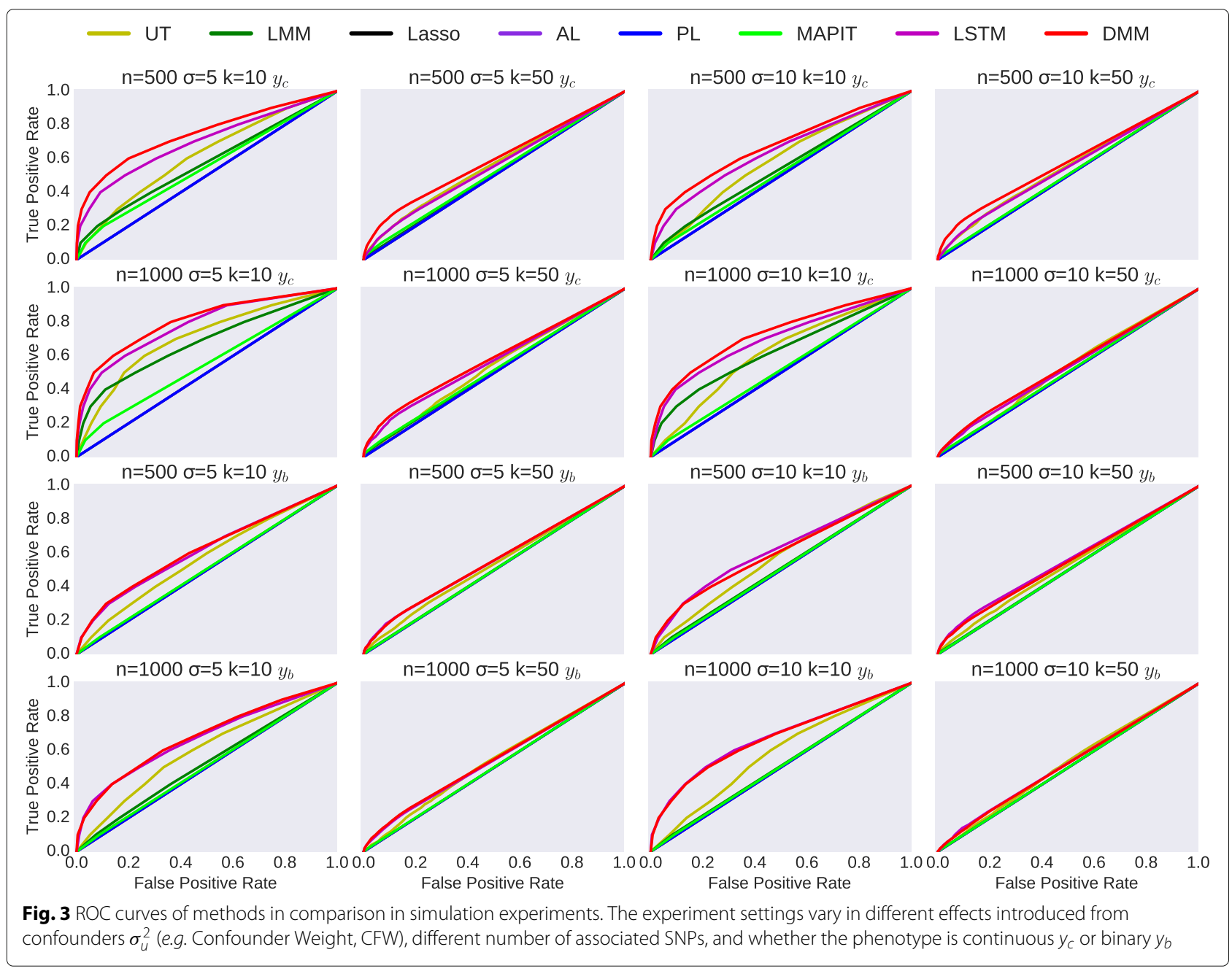


Table 2 Average AUC value for different methods with different settings on Binary data (B) and Continuous Data (C)

\begin{tabular}{llllllllllll}
\hline Pheno & $n$ & $\sigma$ & $k$ & LSTM & DMM & LMM & UT & LASSO & AL & PL & MAPIT \\
\hline C & 500 & 5 & 10 & 0.68 & 0.73 & 0.57 & 0.58 & 0.50 & 0.50 & 0.50 & 0.56 \\
C & 500 & 5 & 50 & 0.54 & 0.58 & 0.51 & 0.55 & 0.50 & 0.50 & 0.50 & 0.51 \\
C & 500 & 10 & 10 & 0.62 & 0.66 & 0.54 & 0.54 & 0.50 & 0.50 & 0.50 & 0.54 \\
C & 500 & 10 & 50 & 0.54 & 0.58 & 0.51 & 0.54 & 0.50 & 0.50 & 0.50 & 0.51 \\
C & 1000 & 5 & 10 & 0.77 & 0.80 & 0.68 & 0.67 & 0.50 & 0.50 & 0.50 & 0.53 \\
C & 1000 & 5 & 50 & 0.56 & 0.58 & 0.51 & 0.52 & 0.50 & 0.50 & 0.50 & 0.52 \\
C & 1000 & 10 & 10 & 0.68 & 0.71 & 0.63 & 0.57 & 0.50 & 0.50 & 0.50 & 0.51 \\
C & 1000 & 10 & 50 & 0.52 & 0.53 & 0.51 & 0.51 & 0.50 & 0.50 & 0.50 & 0.53 \\
B & 500 & 5 & 10 & 0.59 & 0.60 & 0.51 & 0.52 & 0.50 & 0.50 & 0.50 & 0.51 \\
B & 500 & 5 & 50 & 0.55 & 0.55 & 0.51 & 0.52 & 0.50 & 0.50 & 0.50 & 0.50 \\
B & 500 & 10 & 10 & 0.65 & 0.66 & 0.52 & 0.57 & 0.50 & 0.50 & 0.50 & 0.51 \\
B & 500 & 10 & 50 & 0.53 & 0.54 & 0.51 & 0.52 & 0.50 & 0.50 & 0.50 & 0.50 \\
B & 1000 & 5 & 10 & 0.59 & 0.58 & 0.51 & 0.53 & 0.50 & 0.50 & 0.50 & 0.51 \\
B & 1000 & 5 & 50 & 0.55 & 0.54 & 0.51 & 0.52 & 0.50 & 0.50 & 0.50 & 0.51 \\
B & 1000 & 10 & 10 & 0.66 & 0.65 & 0.51 & 0.54 & 0.50 & 0.50 & 0.50 & 0.51 \\
B & 1000 & 10 & 50 & 0.52 & 0.52 & 0.50 & 0.51 & 0.50 & 0.50 & 0.50 & 0.50 \\
\hline
\end{tabular}

confounding factor correction. Interestingly, there are not many differences between the LMM method and Wald Testing method, which is probably due to the fact that these two methods' lack of power in identifying the associated signals from arbitrary interaction of the data.

For the binary phenotype case, DMM does not have a clear advantage over just the LSTM, which is related to the known difficulties in the mixed model for correcting the confounding factors in binary data [36].

\section{Ability in confounding factor correction}

In addition to evaluation of end performance of DMM, we continue to investigate the internal working mechanism of DMM. Figure 4 shows how both modules of DMM fit the

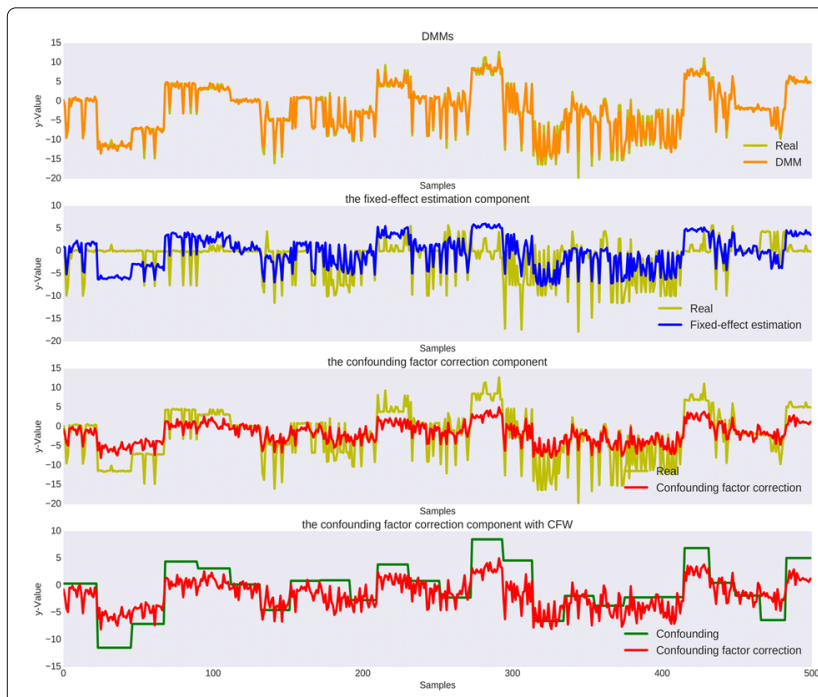

(a)

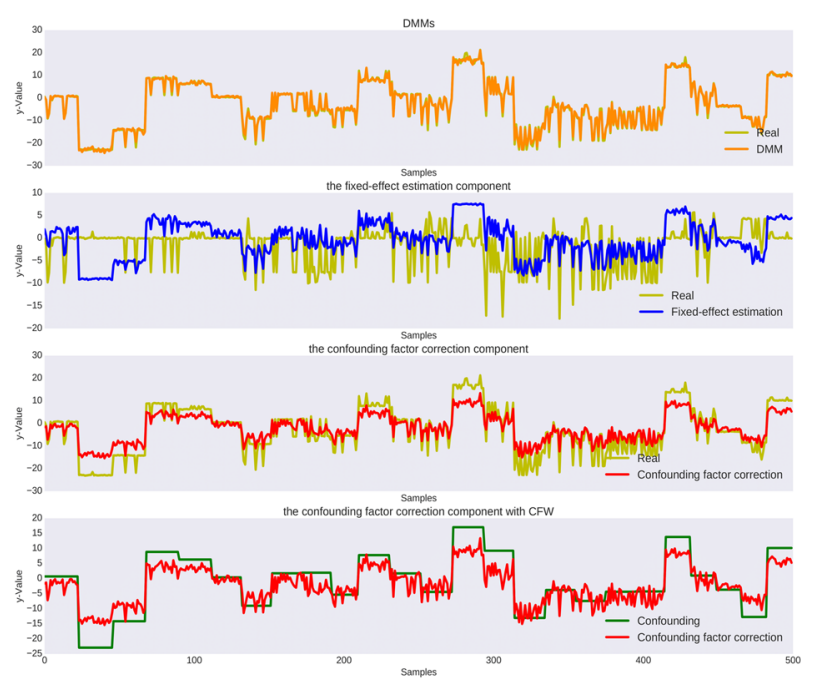

(b)

Fig. 4 Illustration of internal working pattern of DMM. X-axis shows 500 samples and $y$-axis shows the phenotype. For each figure, there are 4 sub-figures. The first one shows how the prediction by DMM (orange) fits the true phenotype (yellow). The second shows how the fixed-effect estimation component (blue) fits the phenotype (yellow). The third one shows the how the confounding factor correction component (red) fits the phenotype (yellow), and the fourth one shows how the confounding factor correction component (red) fits the confounding effects (green). (a) and (b) are two sets of visualizations of the simulation experiments with two different random seeds 
data. With two examples under different setting of confounding factor weight $\sigma$, but same setting of $n=500$, $k=10$, and continuous phenotype, we plot the phenotype across 500 samples, and the prediction made by DMM, the selector, the corrector, and we also plot how the corrector fits the confounding factor curve.

As we can see from both figures in Fig. 4, DMM fits the phenotype very well, and we can barely see the differences between these two curves. Further, with the $2^{\text {nd }}$ and $3^{\text {rd }}$ rows, we can see that neither the selector nor the corrector can predict the phenotype well by itself. At the last row, we can see that the corrector tends to capture the pattern of confounding signals, although there are still gaps between what the corrector fits and the genuine confounding signals. Also, we can observe that, when confounding signals are stronger, the corrector can fit the confounding signals better. These curves verified our design rationale of the DMM: the corrector aims to fit the population level confounding signals, while the selector fits in the residual signals to pinpoint the associated genetic variants.

\section{Application to Alzheimer's Disease}

As previous studies indicated the existence of epistasis in Alzheimer's disease [45], we apply our DMM method to further reveal the genetic architecture of Alzheimer's disease given the success of our method in simulation data.

We combine two different Alzheimer's Disease data sets to increase the sample size. The first one is the AD data provided by Alzheimer's Disease Neuroimaging Initiative (ADNI). We only inspect the individuals that are diagnosed with $\mathrm{AD}$ or Normal in their last visit without considering the patients diagnosed with MCI (mild cognitive impairment). There are 477 individuals. The second one is the late-onset AD dataset provided by Harvard Brain Tissue Resource Center and Merck Research Laboratories [46]. The genotype data were generated from
540 patients in an AD cohort matched for age, gender, and post mortem interval, and consists of the measurements for about 500,000 SNPs. The missing values are imputed as the mode of the corresponding SNPs. For both data sets, we only consider the SNPs that reside proteincoding exons according to GENCODE [47]. We further exclude the SNPs on X-chromosome following suggestions of a previous study [48]. There are 6970 SNPs in the experiment.

\section{Results}

We test the methods on this real data set and apply the models to identify the top 20 SNPs. We report these 20 SNPs in Table 3, where we also list the gene that these SNPs reside in according to GENCODE [47].

Due to the difficulties in verifying epistasis results, we mainly discuss the results reported in Table 3 . However, although most other GWA studies that verify their results through comparison to GWAS Catalog [49], our results are not directly comparable there because most findings in GWAS Catalog are conducted through univariate testing methods. Therefore, we do not expect most of our identified SNPs appear in the GWAS Catalog, which creates a challenge in verifying these reported SNPs. As a result, instead of matching these identified SNPs with GWAS Catalog database for verification, we validate these SNPs through the literature search. Because the community is still learning the functionalities of every single SNP, we study the genes these SNPs reside in as a verification of the genuineness of our discoveries. However, one should be aware that although many pieces of evidence will be presented in the following paragraphs, the evidence only directly supports the relationship between the gene these SNPs reside in and the phenotype, and indirectly serves as the verification that our discovered SNPs are authentic. To the best of our knowledge, this literature-search methodology is the best we can do due to the goal of our proposed model.

Table 3 Top 20 SNPs reported by the Deep Mixed Model that are associated with Alzheimer's disease

\begin{tabular}{llllllllll}
\hline Rank & SNP & Chr & Chr. Position & Gene & Rank & SNP & Chr & Chr. Position & Gene \\
\hline 1 & rs2360982 & 14 & 75764629 & TTLL5 & 11 & rs7310543 & 12 & 69574742 & FRS2 \\
2 & rs4238773 & 16 & 53597938 & RPGRIP1L & 12 & rs4889798 & 17 & 75499805 & TMEM94 \\
3 & rs2424641 & 20 & 24665866 & SYNDIG1 & 13 & rs7959720 & 12 & 27333098 & ARNTL2 \\
4 & rs664866 & 9 & 137077808 & UAP1L1 & 14 & rs7036626 & 9 & 116425812 & ASTN2 \\
5 & rs6706169 & 2 & 165989377 & SCN1A & 15 & rs685417 & 13 & 32511131 & N4BP2L2 \\
6 & rs7149337 & 14 & 50778766 & NIN & 16 & rs405281 & 7 & 150693280 & GIMAP2 \\
7 & rs12881259 & 14 & 90863056 & RPS6KA5 & 17 & rs10876394 & 12 & 51686444 & SCN8A \\
8 & rs12329001 & 2 & 65270990 & ACTR2 & 18 & rs7639223 & 3 & 40260163 & MYRIP \\
9 & rs13242458 & 7 & 99533066 & ZKSCAN5 & 19 & rs12488539 & 3 & 57561864 & PDE12 \\
10 & rs13063312 & 3 & 48636988 & CELSR3 & 20 & rs10402233 & 19 & 40472690 & SPTBN4 \\
\hline
\end{tabular}


Several of these genes have been previously reported to be directly related to Alzheimer's disease. The $5^{\text {th }}$ SNP resides in the gene SCN1A. SCN1A is reported to affect the neural activity of the aging brain [50]. The $10^{\text {th }} \mathrm{SNP}$ resides in the gene CELSR3, which is related to brain development, learning and memory behavior processes in aging mice [51]. The $13^{\text {th }}$ SNP lies in the gene ARNTL2, which has been reported to be associated with Alzheimer disease in Chinese population [52], although the report focused on another SNP within the gene. The $17^{\text {th }}$ SNP resides in the gene $S C N 8 A$, which is one of the few genes that have been reported to be associated with Alzheimer's disease through pathway analysis in mouse model [53]. The $18^{\text {th }}$ SNP resides in gene MYRIP, which is also repoted to be related with Alzheimer's disease [54]. The $20^{\text {th }}$ SNP lies in the gene SPTBN4, which is also reported as a target gene from independent study on other data sets in through DNA methylation map [55].

Several other genes that have not been reported to be directly related to Alzheimer's disease also function in the cognitive activities. For example, the $8^{\text {th }}$ SNP resides in the gene ACTR2, which is identified to be associated with language impairment through copy number analysis [56]. The $12^{\text {th }}$ SNP resides in the gene TEME94, whose variants are associated with neurodevelopmental delay [57]. The $14^{\text {th }}$ SNP lies in the gene ASTN2, which is involved in the neural development [58].

To sum up, these verifications suggest that our identified SNPs and the combinations, although explorative, may reveal some new understandings of Alzheimer's disease. These results also suggest the effectiveness of DMM in identifying the SNPs that contribute to a phenotype with an arbitrarily high order manner.

\section{Discussion}

We also noticed some limitations of our method, for example, the scalability of our method is limited by the memory the GPU. With a modern GPU, our method can only scale up to around 10k SNPs with our current setting. However, as our method only requires a few epoch on the real-world data, a direct fix will be to run our method on CPU clusters instead.

\section{Conclusions}

Following the recent popularity deep learning gains in genetic applications [59], in this paper, we take advantage of the universal approximation property of neural network to build a method that can model the epistasis with arbitrary order of interaction without explicit identifying the combination of SNPs. We built a fixed-effect estimation component that mainly consists of an LSTM, which is well-known for its ability in extracting signals from sequential data. This component is used to identify the associated genetic variants from data. Further, to help eliminate the signals from confounding factors before fixed-effect estimation, we also introduce a confounding factor correction component (a CNN) that helps to remove the effects raised by factors such as population stratification.

Through simulations, we verify the superior performance of our methods over existing methods with simulated data with high-order interaction of SNPs. We further apply our method to Alzheimer's disease data sets and report the SNPs our method filters (and combinations identified later by testing methods). Many of these findings, although explorative, are supported by our literature search verification, thus may reveal some new understandings of Alzheimer's disease.

\section{Abbreviations}

AD: Alzheimer's disease; DMM: Deep mixed model; GWAS: Genome wide association studies; LMM: Linear mixed model; MAF: Minor allele frequency; SNP: Single nucleotide polymorphism

\section{Acknowledgements}

Data collection and sharing for this project were funded by the Alzheimer's Disease Neuroimaging Initiative (ADNI) (National Institutes of Health Grant U01 AG024904). The ADNI is funded by the National Institute on Aging and the National Institute of Biomedical Imaging and Bioengineering and through generous contributions from the following: Abbott; the Alzheimer's

Association; the Alzheimer's Drug Discovery Foundation; Amorfix Life Sciences, Ltd.; AstraZeneca; Bayer HealthCare; BioClinica, Inc.; Biogen Idec, Inc.; Bristol-Myers Squibb Co.; Eisai, Inc.; Elan Pharmaceuticals, Inc.; Eli Lilly and Co.; F. Hoffmann-La Roche, Ltd., and its affiliated company Genentech, Inc.; GE Healthcare; Innogenetics, N.V.; IXICO, Ltd.; Janssen Alzheimer Immunotherapy Research \& Development, LLC.; Johnson \& Johnson Pharmaceutical Research \& Development, LLC.; Medpace, Inc.; Merck \& Co., Inc.; Meso Scale Diagnostics, LLC.; Novartis Pharmaceuticals Corp.; Pfizer, Inc.; Servier; Synarc, Inc.; Takeda Pharmaceutical Co. The Canadian Institutes of Health Research is providing funds to support the ADNI clinical sites in Canada. Private sector contributions are facilitated by the Foundation for the National Institutes of Health (www.fnih.org). The grantee organization is the Northern California Institute for Research and Education, and the study is coordinated by the Alzheimer's Disease Cooperative Study at the University of California, San Diego. ADNI data are disseminated by the Laboratory for Neuro Imaging at the University of California, Los Angeles.

\section{About this supplement}

This article has been published as part of BMC Bioinformatics Volume 20 Supplement 23, 2019: Proceedings of the Joint International GIW \& ABACBS-2019 Conference: bioinformatics. The full contents of the supplement are available online at https://bmcbioinformatics.biomedcentral.com/articles/

supplements/volume-20-supplement-23.

\section{Authors' contributions}

HW proposed the idea, conducted the experiment on Alzheimer's disease and wrote the manuscript. TY and JY improved the idea and conducted the simulation experiments. WW and EPX read and wrote the manuscript. All authors read and approved of the final manuscript.

\section{Funding}

Publication costs are funded and supported by the Department of Defense under Contract No. FA8721-05-C-0003 with Carnegie Mellon University for the operation of the Software Engineering Institute, a federally funded research and development center. This work is also supported by the National Institutes of Health grants R01-GM093156 and P30-DA035778.

\section{Availability of data and materials}

The implementation and datasets used and analysed during the study are available from the corresponding author on reasonable request. 


\section{Ethics approval and consent to participate}

Not applicable.

\section{Consent for publication}

Not applicable.

\section{Competing interests}

The authors declare that they have no competing interests.

\section{Author details}

${ }^{1}$ Language Technologies Institute, School of Computer Science, Carnegie Mellon University, Pittsburgh, PA, USA. ${ }^{2}$ Language Technologies Institute, School of Computer Science, Carnegie Mellon University, Pittsburgh, PA, USA ${ }^{3}$ Department of Electrical and Computer Engineering, Rice University, Houston, TX, USA. ${ }^{4}$ Computational Biology Department, School of Computer Science, Carnegie Mellon University, Pittsburgh, PA, USA. ${ }^{5}$ Machine Learning Department, School of Computer Science, Carnegie Mellon University, Pittsburgh, PA, USA.

\section{Received: 16 November 2019 Accepted: 2 December 2019} Published: 27 December 2019

\section{References}

1. Kang HM, Zaitlen NA, Wade CM, Kirby A, Heckerman D, Daly MJ, Eskin E. Efficient control of population structure in model organism association mapping. Genetics. 2008;178(3):1709-23.

2. Zhang Z, Ersoz E, Lai C-Q, Todhunter RJ, Tiwari HK, Gore MA, Bradbury PJ, Yu J, Arnett DK, Ordovas JM, et al. Mixed linear model approach adapted for genome-wide association studies. Nat Genet. 2010;42(4): 355-60.

3. Tucker G, Price AL, Berger B. Improving the power of gwas and avoiding confounding from population stratification with pc-select. Genetics. 2014;197(3):1045-9.

4. Hayeck TJ, Zaitlen NA, Loh P-R, Vilhjalmsson B, Pollack S, Gusev A, Yang J, Chen G-B, Goddard ME, Visscher PM, et al. Mixed model with correction for case-control ascertainment increases association power. Am J Hum Genet. 2015;96(5):720-30.

5. Zhong S, Jiang D, McPeek MS. Ceramic: Case-control association testing in samples with related individuals, based on retrospective mixed model analysis with adjustment for covariates. PLoS Genet. 2016;12(10):1006329.

6. Ogutu JO, Schulz-Streeck T, Piepho H-P. Genomic selection using regularized linear regression models: ridge regression, lasso, elastic net and their extensions. In: BMC Proceedings, vol. 6; 2012. p. 1. BioMed Central. https://doi.org/10.1186/1753-6561-6-s2-s10.

7. Zou H. The adaptive lasso and its oracle properties. J Am Stat Assoc. 2006;101(476):1418-29.

8. Wang $H$, Lengerich BJ, Aragam B, Xing EP, Stegle O. Precision lasso: Accounting for correlations and linear dependencies in high-dimensional genomic data. Bioinformatics. 2018;1:7.

9. Rakitsch B, Lippert C, Stegle O, Borgwardt K. A lasso multi-marker mixed model for association mapping with population structure correction. Bioinformatics. 2012;29(2):206-14.

10. Wang H, Aragam B, Xing EP. Variable selection in heterogeneous datasets: A truncated-rank sparse linear mixed model with applications to genome-wide association studies. Methods. 2017. https://doi.org/10. 1109/bibm.2017.8217687.

11. Mäki-Tanila A, Hill WG. Influence of gene interaction on complex trait variation with multi-locus models. Genetics. 2014114. https://doi.org/10 1534/genetics.114.165282.

12. Eichler EE, Flint J, Gibson G, Kong A, Leal SM, Moore JH, Nadeau JH. Missing heritability and strategies for finding the underlying causes of complex disease. Nat Rev Genet. 2010;11(6):446.

13. Gibson G. Hints of hidden heritability in gwas. Nat Genet. 2010;42(7):558

14. Zuk O, Hechter E, Sunyaev SR, Lander ES. The mystery of missing heritability: Genetic interactions create phantom heritability. Proc Natl Acad Sci. 2012;109(4):1193-8.

15. Wei W-H, Hemani G, Haley CS. Detecting epistasis in human complex traits. Nat Rev Genet. 2014;15(11):722.

16. Strange A, Capon F, Spencer CC, Knight J, Weale ME, Allen MH, Barton A, Band G, Bellenguez C, Bergboer JG, et al. A genome-wide association study identifies new psoriasis susceptibility loci and an interaction between hla-c and erap1. Nat Genet. 2010;42(11):985

17. Evans DM, Spencer CC, Pointon JJ, Su Z, Harvey D, Kochan G, Oppermann U, Dilthey A, Pirinen M, Stone MA, et al. Interaction between erap1 and hla-b27 in ankylosing spondylitis implicates peptide handling in the mechanism for hla-b27 in disease susceptibility. Nat Genet. 2011;43(8):761

18. Hemani G, Shakhbazov K, Westra H-J, Esko T, Henders AK, McRae AF, Yang J, Gibson G, Martin NG, Metspalu A, et al. Detection and replication of epistasis influencing transcription in humans. Nature. 2014;508(7495):249

19. Zhang $X$, Huang S, Zou F, Wang W. Team: efficient two-locus epistasis tests in human genome-wide association study. Bioinformatics. 2010;26(12):217-27.

20. Schüpbach T, Xenarios I, Bergmann S, Kapur K. Fastepistasis: a high performance computing solution for quantitative trait epistasis. Bioinformatics. 2010;26(11):1468-9.

21. Liu Y, Xu H, Chen S, Chen X, Zhang Z, Zhu Z, Qin X, Hu L, Zhu J, Zhao $G-P$, et al. Genome-wide interaction-based association analysis identified multiple new susceptibility loci for common diseases. PLoS Genet. 2011;7(3):1001338.

22. Gyenesei A, Moody J, Semple CA, Haley CS, Wei W-H. High-throughput analysis of epistasis in genome-wide association studies with biforce. Bioinformatics. 2012;28(15):1957-64.

23. Lippert C, Listgarten J, Davidson Rl, Baxter J, Poon H, Kadie CM, Heckerman D. An exhaustive epistatic snp association analysis on expanded wellcome trust data. Sci Rep. 2013;3:1099.

24. Prabhu S, Pe'er I. Ultrafast genome-wide scan for snp-snp interactions in common complex disease. Genome Res. 2012;22(11):2230-40.

25. Hahn LW, Ritchie MD, Moore JH. Multifactor dimensionality reduction software for detecting gene-gene and gene-environment interactions. Bioinformatics. 2003;19(3):376-82.

26. Nunkesser R, Bernholt T, Schwender H, Ickstadt K, Wegener I. Detecting high-order interactions of single nucleotide polymorphisms using genetic programming. Bioinformatics. 2007;23(24):3280-8.

27. Emily M, Mailund T, Hein J, Schauser L, Schierup MH. Using biological networks to search for interacting loci in genome-wide association studies. Eur J Hum Genet. 2009;17(10):1231.

28. Yang P, Ho JW, Zomaya AY, Zhou BB. A genetic ensemble approach for gene-gene interaction identification. BMC Bioinformatics. 2010;11(1):524

29. Kam-Thong T, Czamara D, Tsuda K, Borgwardt K, Lewis CM Erhardt-Lehmann A, Hemmer B, Rieckmann P, Daake M, Weber F, et al. Epiblaster-fast exhaustive two-locus epistasis detection strategy using graphical processing units. Eur J Hum Genet. 2011;19(4):465.

30. Goudey B, Rawlinson D, Wang Q, Shi F, Ferra H, Campbell RM, Stern L, Inouye MT, Ong CS, Kowalczyk A. Gwis-model-free, fast and exhaustive search for epistatic interactions in case-control gwas. BMC Genomics. 2013;14(3):10.

31. Leem S, Jeong H.-h., Lee J, Wee K, Sohn K-A. Fast detection of high-order epistatic interactions in genome-wide association studies using information theoretic measure. Comput Biol Chem. 2014;50:19-28.

32. Goudey B, Abedini M, Hopper JL, Inouye M, Makalic E, Schmidt DF, Wagner J, Zhou Z, Zobel J, Reumann M. High performance computing enabling exhaustive analysis of higher order single nucleotide polymorphism interaction in genome wide association studies. Health Inform Sci Syst. 2015;3(S1):3.

33. Crawford L, Zeng P, Mukherjee S, Zhou X. Detecting epistasis with the marginal epistasis test in genetic mapping studies of quantitative traits. PLoS Genet. 2017;13(7):1006869.

34. Wang H, Raj B, Xing EP. On the origin of deep learning. 2017. arXiv preprint arXiv:1702.07800

35. Mishkin D, Sergievskiy N, Matas J. Systematic evaluation of cnn advances on the imagenet. 2016. arXiv preprint arXiv:1606.02228.

36. Yang J, Zaitlen NA, Goddard ME, Visscher PM, Price AL. Advantages and pitfalls in the application of mixed-model association methods. Nat Genet. 2014;46(2):100

37. LiY, Chen C-Y, Wasserman WW. Deep feature selection: Theory and application to identify enhancers and promoters. In: International Conference on Research in Computational Molecular Biology. Springer; 2015. p. 205-17. 
38. Prechelt L. Early stopping-but when? In: Neural Networks: Tricks of the Trade. Springer; 1998. p. 55-69. https://doi.org/10.1007/3-540-494308_3.

39. Caruana R, Lawrence S, Giles CL. Overfitting in neural nets: Backpropagation, conjugate gradient, and early stopping. In: Advances in Neural Information Processing Systems; 2001. p. 402-8. https://doi.org/ 10.1109/ijenn.2000.857823.

40. Benjamini Y, Hochberg Y. Controlling the false discovery rate: a practical and powerful approach to multiple testing. J R Stat Soc Ser B (Methodol). 1995289-300. https://doi.org/10.1111/j.2517-6161.1995.tb02031.x.

41. Tibshirani R. Regression shrinkage and selection via the lasso. J R Stat Soc Ser B (Methodol). 1996267-88. https://doi.org/10.1111/j.2517-6161.1996. tb02080.x.

42. Huang J, Ma S, Zhang C-H. Adaptive lasso for sparse high-dimensional regression models. Stat Sin. 2008;1603-18.

43. Peng B, Kimmel M. simupop: a forward-time population genetics simulation environment. Bioinformatics. 2005;21(18):3686-7.

44. Manolio TA, Collins FS, Cox NJ, Goldstein DB, Hindorff LA, Hunter DJ, McCarthy MI, Ramos EM, Cardon LR, Chakravarti A, et al. Finding the missing heritability of complex diseases. Nature. 2009;461(7265):747-53.

45. Combarros O, Cortina-Borja M, Smith AD, Lehmann DJ. Epistasis in sporadic alzheimer's disease. Neurobiol Aging. 2009;30(9):1333-49.

46. Zhang B, Gaiteri C, Bodea L-G, Wang Z, McElwee J, Podtelezhnikov AA, Zhang C, Xie T, Tran L, Dobrin R, et al. Integrated systems approach identifies genetic nodes and networks in late-onset alzheimer's disease. Cell. 2013;153(3):707-20.

47. Harrow J, Frankish A, Gonzalez JM, Tapanari E, Diekhans M, Kokocinski F, Aken BL, Barrell D, Zadissa A, Searle S, et al. Gencode: the reference human genome annotation for the encode project. Genome Res. 2012;22(9):1760-74

48. Bertram L, Lange C, Mullin K, Parkinson M, Hsiao M, Hogan MF, Schjeide BM, Hooli B, DiVito J, Ionita I, et al. Genome-wide association analysis reveals putative alzheimer's disease susceptibility loci in addition to apoe. Am J Hum Genet. 2008;83(5):623-32.

49. Welter D, MacArthur J, Morales J, Burdett T, Hall P, Junkins H, Klemm A, Flicek P, Manolio T, Hindorff L, et al. The nhgri gwas catalog, a curated resource of snp-trait associations. Nucleic Acids Res. 2013;42(D1):1001-6.

50. Meier S, Demirakca T, Brusniak W, Wolf I, Liebsch K, Tunc-Skarka N, Nieratschker V, Witt SH, Matthäus F, Ende G, et al. Scn1a affects brain structure and the neural activity of the aging brain. Biol Psychiatry. 2012;72(8):677-83.

51. Cheong L-Z, Sun T, Li Y, Zhou J, Lu C, Li Y, Huang Z, Su X. Dietary krill oil enhances neurocognitive functions and modulates proteomic changes in brain tissues of d-galactose induced aging mice. Food Funct. 2017;8(5):2038-45.

52. Qing-Xiu L, Chang-Quan H, Qian C, Xue-Mei Z, Xiu-Ying H, Song-Bing L. The polymorphism of arntl2 (bmal2) gene rs $2306074 \mathrm{C} i \mathrm{t}$ is associated with susceptibility of alzheimer disease in chinese population. Neurol Sci. 2014;35(11):1743-7.

53. Hsu W-CJ, Wildburger NC, Haidacher SJ, Nenov MN, Folorunso O, Singh AK, Chesson BC, Franklin WF, Cortez I, Sadygov RG, et al. Ppargamma agonists rescue increased phosphorylation of fgf14 at s226 in the tg2576 mouse model of alzheimer's disease. Exp Neurol. 2017;295:1-17.

54. Zhang L, Ju X, Cheng Y, Guo X, Wen T. Identifying tmem59 related gene regulatory network of mouse neural stem cell from a compendium of expression profiles. BMC Syst Biol. 2011;5(1):152.

55. Sanchez-Mut JV, Aso E, Panayotis N, Lott I, Dierssen M, Rabano A, Urdinguio RG, Fernandez AF, Astudillo A, Martin-Subero Jl, et al. Dna methylation map of mouse and human brain identifies target genes in alzheimer's disease. Brain. 2013;136(10):3018-27.

56. Simpson NH, Ceroni F, Reader RH, Covill LE, Knight JC, Nudel R, Monaco A, Simonoff E, Bolton P, Pickles A, et al. Genome-wide analysis identifies a role for common copy number variants in specific language impairment. Eur J Hum Genet. 2015;23(10):1370.

57. Stephen J, Maddirevula S, Nampoothiri S, Burke JD, Herzog M, Shukla A, Steindl K, Eskin A, Patil SJ, Joset P, et al. Bi-allelic tmem94 truncating variants are associated with neurodevelopmental delay, congenital heart defects, and distinct facial dysmorphism. Am J Hum Genet. 2018;103(6): 948-67.
58. Ni T, Harlos K, Gilbert R. Structure of astrotactin-2: a conserved vertebrate-specific and perforin-like membrane protein involved in neuronal development. Open Biol. 2016;6(5):160053.

59. Yue T, Wang H. Deep learning for genomics: A concise overview. 2018. arXiv preprint arXiv:1802.00810.

\section{Publisher's Note}

Springer Nature remains neutral with regard to jurisdictional claims in published maps and institutional affiliations.
Ready to submit your research? Choose BMC and benefit from:

- fast, convenient online submission

- thorough peer review by experienced researchers in your field

- rapid publication on acceptance

- support for research data, including large and complex data types

- gold Open Access which fosters wider collaboration and increased citations

- maximum visibility for your research: over $100 \mathrm{M}$ website views per year

At BMC, research is always in progress.

Learn more biomedcentral.com/submissions 